\title{
Surface studies on as-grown (111) faces of sodium bromate crystals
}

\author{
K KISHAN RAO* and V SURENDER ${ }^{\dagger}$ \\ Department of Physics, Kakatiya University, Warangal 506 009, India \\ ${ }^{\dagger}$ Government Junior College, Warangal 506 001, India
}

MS received 13 November 2000; revised 17 September 2001

\begin{abstract}
Single crystals of sodium bromate are grown at various supersaturations ranging from $3 \%$ to $8 \%$. Surface studies have been carried out on as-grown and etched (111) faces of these crystals. Typical and systematically oriented growth hillocks are observed almost on all the faces. Further dislocation studies are made to understand the growth history of these crystals. These studies suggest that the crystals grow by 2D-growth mechanism. In addition to this, studies are also conducted on the formation of overgrowths and inclusions in these crystals.
\end{abstract}

Keywords. Crystal growth; sodium bromate; surface studies; growth mechanism.

\section{Introduction}

There are several models of crystal growth (Kossel 1927; Stransky 1928; Frank 1949) and each crystal grows by one of these mechanisms. One method of studying crystal growth mechanism is to compare measured crystal growth rates with theoretically predicted growth rates (Bennema 1965). A complementary approach is to study the features on free and etched surfaces of crystals. Patterns observed on surfaces like spirals, hillocks, slip pattern, etc yield considerable information on the growth process in the crystals (Joshi and Vagh 1967).

Sodium bromate is a cubic crystal (point group 23). It grows from aqueous solution in the form of pyramids bounded by [111] faces. A study of the growth mechanism in these crystals has been made earlier by the present authors (Surender and Kishan Rao 1995) by measuring the growth rates as a function of supersaturation. The evidence was in favour of $2 \mathrm{D}$-growth mechanism. The purpose of the present paper is to report complementary evidence on growth mechanism by studying features on free and etched surfaces of sodium bromate crystals. In addition, overgrowths and inclusions have also been studied.

\section{Experimental}

Single crystals of sodium bromate were grown at various supersaturations ranging from $3 \%$ to $8 \%$ at $30^{\circ} \mathrm{C}$. The details of growth method was published earlier (Surender and Kishan Rao 1995). The crystals grown were gently dried and immediately transferred to Meopta microscope

\footnotetext{
*Author for correspondence
}

to observe the surfaces. The crystals were etched using an etchant consisting of 8 parts of glacial acetic acid, 2 parts of formic acid $+2 \mathrm{mg} / \mathrm{cc}$ of cupric nitrate. To study the etch pit pattern, overgrowths and inclusions, transmission microscopy was employed.

\section{Results and discussion}

\subsection{Features on free and etched surfaces}

The (111) faces of sodium bromate crystals exhibit several surface features such as growth hillocks, overgrowths, inclusions and striations. In the present work, at least sixty crystals grown at various supersaturations ranging from $3 \%$ to $8 \%$ are studied under reflection microscope. Most of these faces have shown growth hillocks. Figure 1a shows randomly distributed but strictly oriented growth hillocks. These hillocks are triangular in shape. Their edges are slightly curved and are oriented parallel to the edges of the crystal faces i.e. along <110> directions. These reflect the three-fold rotational symmetry of the faces. Their density not only varies from crystal to crystal but also from face to face of the same crystal.

It is known that summit of growth pyramids is the centre of initiation of growth layers. Growth of crystal face may start simultaneously from a number of nuclei formed on the face, but all of them may not remain active throughout the growth process. In fact most of the initial growth centres become inactive in a relatively shorter time of the growth process and thus the growth of each face is governed by few number of growth centres. The growth hillocks observed are composed of closed triangular loops, one such hillock is shown in figure $1 \mathrm{~b}$ at higher magnification, the hillocks appear to have formed 
due to piling and spreading of triangular growth layers. The spacing between the successive growth fronts is not the same. It is known to depend on the advancement of growth fronts. Faster the growth rate smaller is the spacing, which in turn depends on the number of molecules absorbed at the steps. Perhaps the reason for discrepancy in spacing of steps could be due to some microscopical disturbance in supersaturation during the growth process.

To probe whether the growth centres are due to dislocations or not, crystal faces are chemically etched by an improved method developed in the laboratory, which facilitates to etch almost at the selective position. It consists of a small tube with pointed tip, after filling with etchant, it is fixed to the microscope socket provided for objectives. After ascertaining the position of the growth
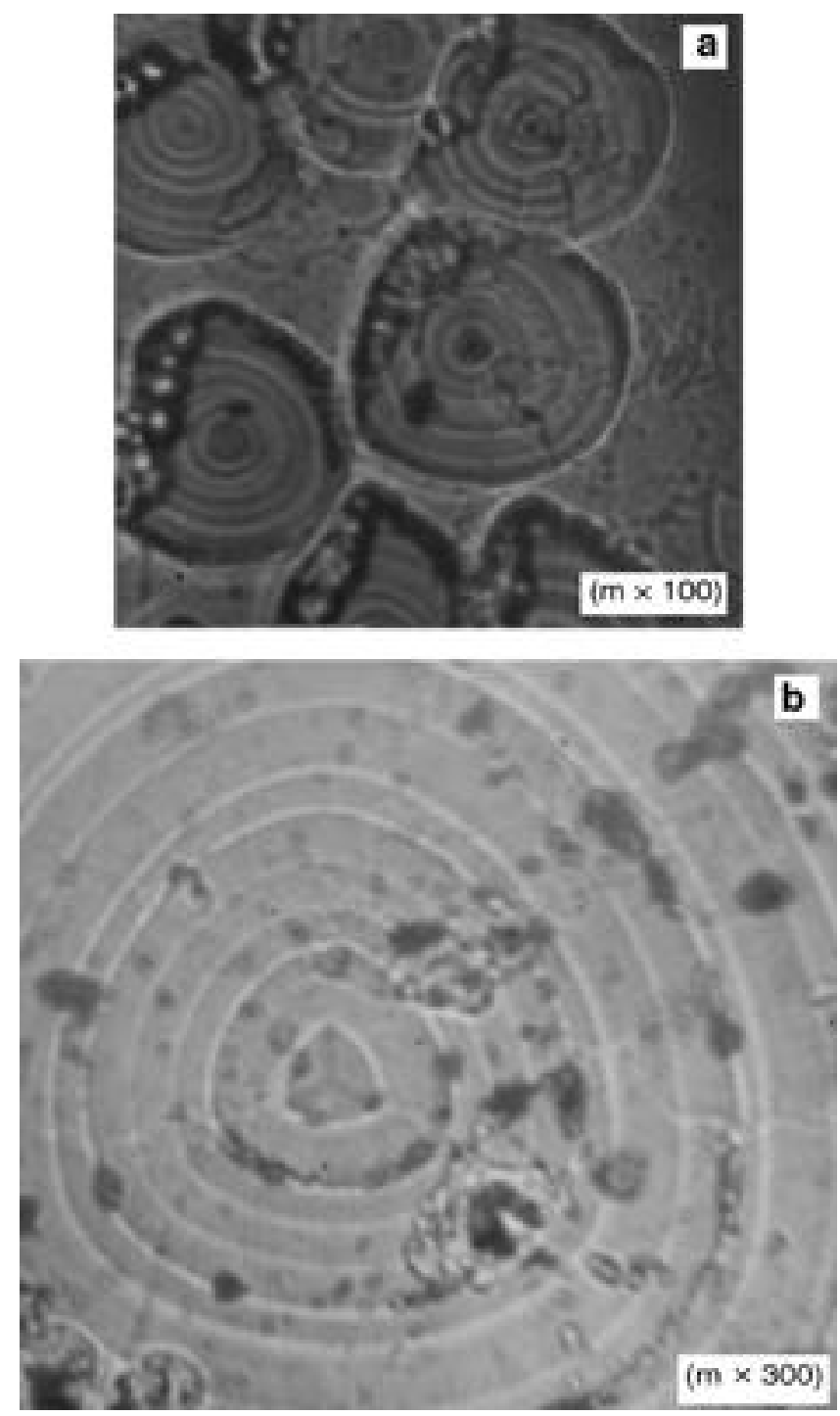

Figure 1. a. Typical growth hillocks on (111) face of sodium bromate crystals and $\mathbf{b}$. one of the growth hillocks under higher magnification. feature at higher magnification, the tube is brought into the selective position by turning the socket. By pressing the tube gently the etchant is released drop by drop at that spot. The etchant is dried on the microscope stage with the help of tissue paper or with hot air blower. The crystal surface is again brought into focus by turning the socket. Figure 2 shows one of the etched regions, the etch pits are triangular in shape, with sides oriented along <110> direction. Similar type of etching experiments have been made at least on twenty crystal faces and it has been observed that no growth centre has turned into an etch pit but a number of etch pits are formed nearby. The present etching studies together with surface studies indicate that the growth hillocks appear to be not due to screw dislocations. These studies further confirm that the growth of (111) faces appear to be due to 2D-growth mechanism. As pointed out by Sangwal (1989), these hillocks can be called as non-dislocation origin growth hillocks, which depend on supersaturation, the shape of the growth cell, size of the crystal face etc.

\subsection{Overgrowth studies}

Some of the (111) faces of sodium bromate crystals show typical overgrowth hillocks formed during the growth, they are either triangular or more or less hexagonal in shape. According to Buckley (1951) overgrowths form due to interruption in the continuity of deposition of material which in turn is due to change in growth conditions. Figure 3 a shows a hexagonal overgrowth and etch pattern on it, the orientation of the etch pits on this overgrowth is slightly different from the orientation of the pits formed on the host crystal surface. Figure $3 \mathrm{~b}$ shows etch pit pattern near the same overgrowth recorded under lower magnification. It can be seen from this photograph that the etch pits formed on the overgrowth are slightly bigger than the pits formed nearby. Perhaps the formation of smaller pits near the overgrowth could be due to fresh

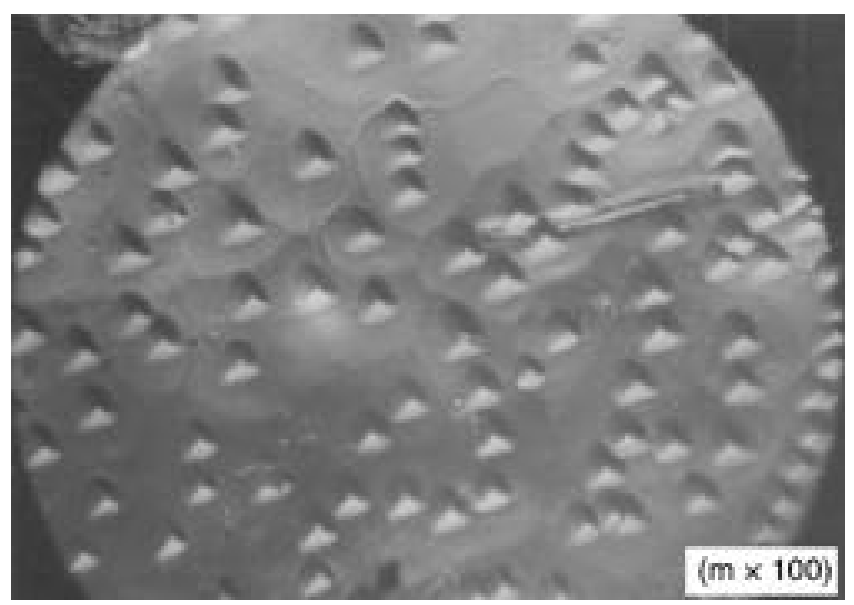

Figure 2. Etch pit pattern on (111) face of sodium bromate. 
dislocations formed due to interaction of the overgrowth with the host surface. The difference in the orientation of etch pits from the host crystal surface suggests that the overgrowth is slightly misoriented with respect to the host crystal surface. Figure $3 \mathrm{c}$ shows the etch pattern around a triangular overgrowth. It is interesting to note that no etch pits are formed on this overgrowth whereas etch pits are formed on the host surface. These results indicate that
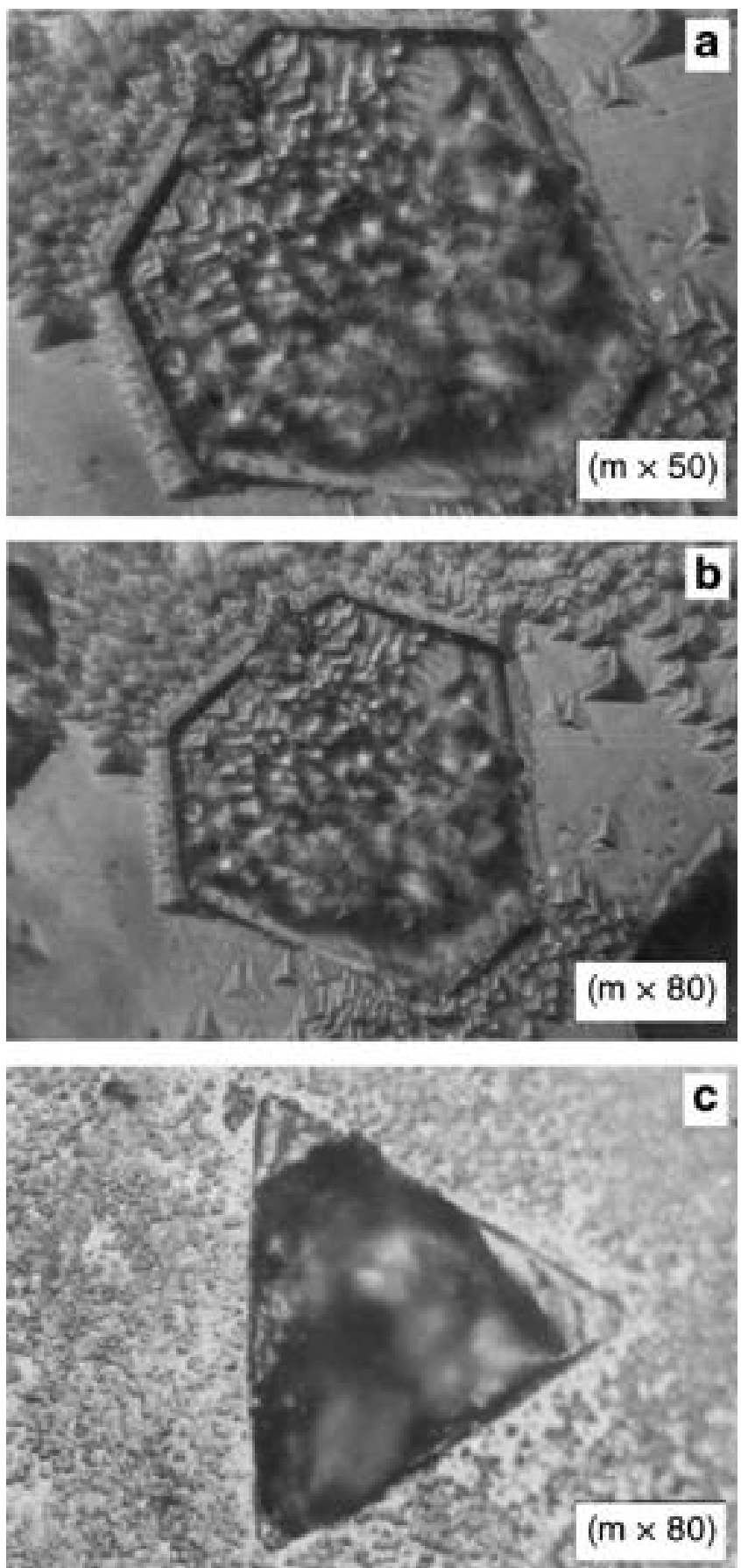

Figure 3. a. Typical hexagonal overgrowth on (111) face showing etch pits also, b. etch pit pattern near the overgrowth and c. etch pit pattern around a triangular overgrowth. hexagonal overgrowths appear to be major (111) faces and triangular overgrowths could be minor (111) faces, because the etchant acts as selective etchant on major (111) faces only but not on minor (111) faces, this is due to non-centrosymmetric nature of the crystals (Kishan Rao and Sirdeshmukh 1983). Figure 4a shows typical
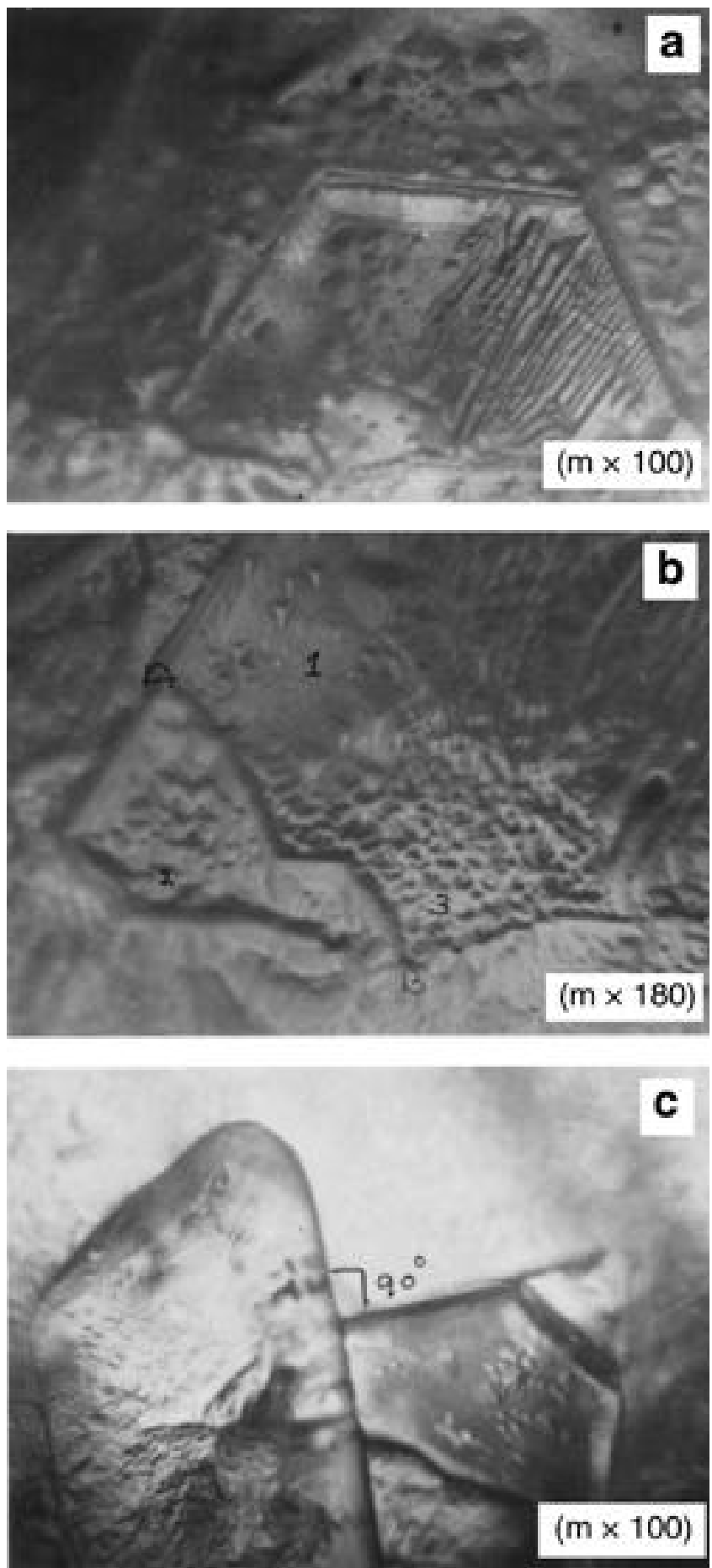

Figure 4. a. Stratigraphical pattern on a overgrowth, b. regions of (4a) after chemical etching and c. microphotograph of typical inclined overgrowths. 

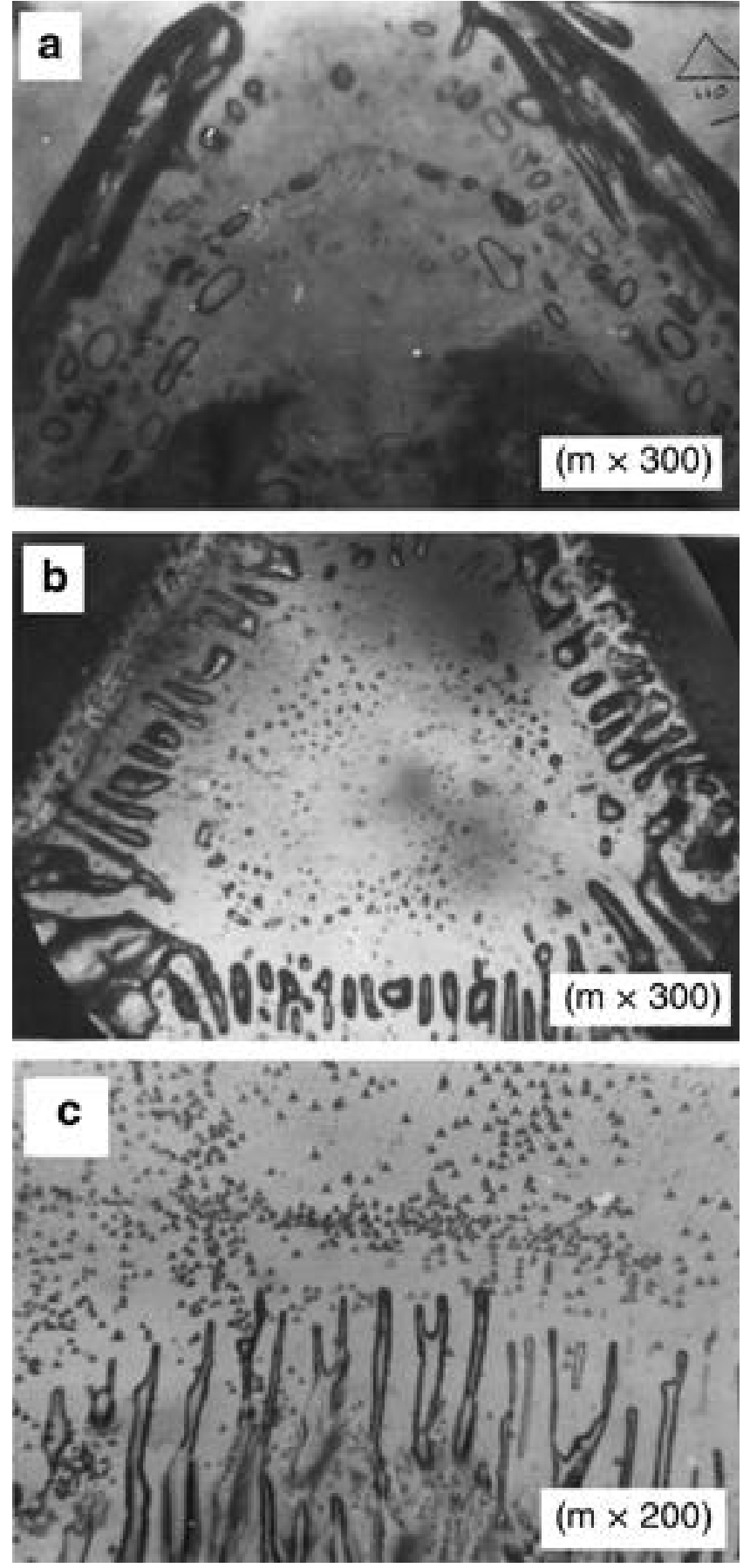

Figure 5. a-b. Microphotograph of inclusions at super saturations $3 \%$ and $5 \%$, respectively, and c. etch pit pattern near the inclusions.

stratigraphical pattern on a overgrowth, they are almost parallel to one of its edges. Figure $4 \mathrm{~b}$ shows the same region after etching and at higher magnification. It shows a typical boundary along $\mathrm{AB}$ and the etch pattern is shown as 1,2 and 3 regions in the photograph.

The orientation of etch pits in region 1 is exactly opposite to etch pits formed in regions 2 and 3. From these observations it can be understood that though the overgrowth appears to be a single overgrowth but it consists of small overgrowths mis-oriented with respect to each other. The formation of striations on the overgrowth indicate that their growth appears to be due to sheetlayer mechanism. Figure 4c shows microphotograph of typical overgrowths inclined with each other at an angle about $90^{\circ}$ (marked on the photograph). The photograph further shows etch pits formed on the overgrowths, their apices point towards the apices of their respective overgrowths.

\subsection{Inclusions}

In the present study attention has also been focused to study the inclusions in the sodium bromate crystals. In general it is observed that almost all the crystals contain inclusions. Figures 5a, b show transmission microphotographs of typical inclusions grown at different supersaturations $3 \%$ and $5 \%$. The inclusions are mostly trapped at the edges i.e. along $\langle 110\rangle$ directions of the crystals. The inclusions are of various shapes and sizes. Some of them are oval shaped and some appear like elongated cylinders. A variety of parameters such as variation in supersaturation during the growth, nonuniform growth rates etc are responsible for the formation of inclusions. The reason for majority of the inclusions getting trapped at the edges could be due to fluctuations in supersaturation near the crystal or due to transition from dissolution to growth. Kitamura et al (1982) pointed out that liquid inclusions getting trapped parallel to the interfaces are due to drastic changes in growth condition. It has been observed that the density of inclusions slightly increases with supersaturation. Figure $5 \mathrm{c}$ shows the etch pattern near the inclusions. The number of etch pits formed near them is less but the etch pits are distributed away from the inclusions. This shows that inclusions may not be acting as preferential sites or source for production of dislocations. Similar observations are made on a number of crystals in the present study.

\section{Conclusions}

(I) Study of triangular growth hillocks and etch pits suggest that the growth of these crystal faces is due to 2Dgrowth mechanism.

(II) Overgrowths formed during the growth are slightly mis-oriented with respect to the host face.

(III) Formation of inclusions could be due to fluctuation in supersaturation, growth rate etc during growth of the crystals.

\section{Acknowledgements}

The authors thank Prof. D B Sirdeshmukh, former Head, Department of Physics, Kakatiya University, Warangal 
for his interest in the work and valuable suggestions. The authors are grateful to the referees for useful suggestions.

\section{References}

Bennema P 1965 The rate of growth of crystals from slightly supersaturated solutions, Ph.D. Thesis, Technical University of Delft, Delft

Buckley H E 1951 Crystal growth (New York: John Wiley \& Sons)

Frank F C 1949 Dis. Faraday Soc. 548
Joshi M S and Vagh A S 1967 Indian J. Pure \& Appl. Phys. 5 318

Kishan Rao K and Sirdeshmukh D B 1983 Cryst. Res. Tech. 18 1125

Kitamura M, Kouchi A, Hosoya S and Sunagawa I 1982 Miner. J. 3119

Kossel W 1927 Nachr. Akad. Wiss. Gottingen. Math. Phys. Klasse. 135

Sangwal K 1989 Prog. Crystal Growth and Charact. (GB) 19189

Stransky I N 1928 Z. Phys. Chem. 136259

Surender V and Kishan Rao K 1995 Bull. Mater. Sci. 18289 